\title{
Study on Polymer Materials Evaluation System for Nano-Imprint Lithography
}

\author{
Atsushi Sekiguchi, Yoshiyuki Kono and Yoshihiko Hirai ${ }^{* 1}$ \\ Litho Tech Japan Corp., 2-6-6 Namiki, Kawaguchi, Saitama 332-0034, Japan \\ ${ }^{*}$ Osaka Prefecture Univ. 1-1 Gakuen-cho, Sakai, Osaka, 599-8531, Japan
}

\begin{abstract}
Numerous methods are available for lithography below the $100 \mathrm{~nm}$ node scale, including $F_{2}, 193 \mathrm{~nm}$ immersion, EB, EUV, and imprint lithography. Among these methods, imprint lithography has attracted significant attention because it does not require expensive exposure equipment. Imprint lithography can be performed by one of two primary methods: the thermal method or the UV curing method.[2] In thermal imprinting, the resin is softened above $T_{g}$ before being formed by a mold. In UV imprinting, a transparent mold is applied to a liquid resin, which is then exposed to UV light for curing. Thermal imprinting requires a pressure of $10 \mathrm{MPa}$ and consumes throughput (to increase and reduce the temperature) time requires time for throughput (i.e., time required to increase and reduce temperatures). In contrast, UV imprinting does not require high pressure, since the resin is basically a viscous liquid and soft enough to be deformed. However, since the resin is in liquid form, the UV imprinting process is sensitive to the flatness of the substrate and mold. Problems of nonuniformity (i.e., interference patterns) have been noted in residual film distribution. In response, we developed what we call the PEP method, which combines the advantages of both thermal and UV imprinting. We have performed various experiments to examine the consequences of the PEP approach. The Pre-Exposure Process method essentially consists of a type of UV imprinting, but one in which the resin is subject to extremely weak exposed prior to the pressing exposed to very weak UV radiation before pressing, which slightly hardens the resist and increases rigidity. The mold is then pressed to shape the resin, followed by the primary exposure. This process allows the resin to maintain softness equivalent to that at or above $T_{g}$ in thermal imprinting, while allowing processing, as in UV imprinting. We also examined the relationship between exposure and crosslinking ratios, using FT-IR equipment with an exposure function, to determine the optimal crosslinking ratio for the PEP method. The results of these examinations are also reported.
\end{abstract}

Keywords: Nano imprint, Pre-exposure, Crosslinking ratio, FT-IR, QCM

\section{Introduction}

Many lithography technologies at or below the $100 \mathrm{~nm}$ node scale are being investigated, including the $F_{2}, 193 \mathrm{~nm}$ immersion, EB, EUV, and Nano imprint methods. Among these choices, nano imprint lithography [1] is attracting significant attention, as it does not require expensive exposure equipment. Imprint lithography can be broadly divided into thermal lithography and UV imprint lithography. In thermal-imprint lithography, the resin is heated to $T_{g}$ or higher so that it is softened, and then patterning is performed by pressing a mold against the resin. In UV imprint lithography, in contrast, liquid resin is exposed to UV light for curing while a transparent mold is pressed against the resin.With thermal-imprint lithography [2-6], there is a high degree of freedom in the selection of materials, as any thermoplastic resins can be used. In addition, as the resin does not melt when it is heated up to or above $T_{g}$, good mold release characteristics are obtained. Furthermore, the uniform thickness distribution of residual films due to the high pressing pressure eliminates problems such as interference patterns. However, it requires a mold pressure of 10 to $30 \mathrm{MPa}$ at or above $\mathrm{T}_{\mathrm{g}}$, and a large-scale pressing mechanism is needed. Low throughput is another problem with thermal-imprint lithography, as it requires a thermal process for heating and cooling of the resin. In contrast, UV imprint lithography [7-9] does not require high 
pressure and a large-scale pressing mechanism, as liquid resin is shaped into the mold. It also does not involve a thermal process, which is another advantage of the method compared with thermalimprint lithography relating to throughput. However, the materials that can be used in the UV imprinting process are limited, as it requires photosetting resin, and the mold release characteristics are poor due to the high tackiness of the resin. Furthermore, the process is sensitive to the parallelism and flatness of the stage and mold, as the resin is in liquid form, and the resulting nonuniform thickness distribution of the residual films causes interference patterns. It has also been pointed out that the diffraction caused by the mold may result in fatal defects, depending on the setting of resist film thickness [10].

To solve these problems, we have developed what we have termed the PEP (Pre-Exposure Process)method, which combines the advantages of both thermal and UV imprinting methods, and have conducted experiments to demonstrate the effects of the PEP method. While the PEP method is essentially a type of UV imprint lithography, the resin is subjected to extremely weak light exposure prior to the pressing operation. The resist is hardened slightly by this process and its rigidity is increased, making it possible to perform UV imprint lithography while maintaining the softness of the resin equivalent to that at or above $T_{g}$ in thermal-imprint lithography. We have investigated the relationship between light exposure and the crosslinking ratio using FT-IR equipment [11] provided with an exposure function to determine the optimal crosslinking ratio in the PEP method, and then verified experimentally the effects of the PEP method on the imprinting process based on the results obtained regarding the relationship.

\section{Imprint Equipment}

Figure 1 shows the imprinting equipment MODEL LTNIP-5000 made by Litho Tech Japan Corporation (hereinafter referred to as "LTJ") that was used in the present experiments for both thermal imprinting and UV imprinting. It is driven by a servo motor and a ball screw, and provides a maximum pressure of $20 \mathrm{MPa}$.(imprint area: 1 square inch) It is equipped with a source of UV light, and the emitted UV light is led to the press stage through optical fibers in order to irradiate the mold. The exposure is controlled arbitrarily by setting conditions on the operation panel. Furthermore, a vacuum chamber is provided to enable both thermal and UV imprinting in a vacuum. Figure 1 (b) shows a scene in which UV exposure is being performed (in practice, the chamber is closed to keep it in a vacuum). The stage on which the wafer is placed is equipped with a simple alignment mechanism for the $\mathrm{X}, \mathrm{Y}, \mathrm{Z}$, and theta directions so that alignment can be performed for UV imprinting. The size of the mold is 1 inch square, and quartz glass molds are used when photo printing or alignment is required. Circular silicon substrates with a diameter of 2 inches or silicon substrates that are $35 \mathrm{~mm}$ square are used. Furthermore, a heating and cooling unit used for the thermal imprinting process is provided with a maximum heating temperature of $250^{\circ} \mathrm{C}$. Water is used as the coolant.

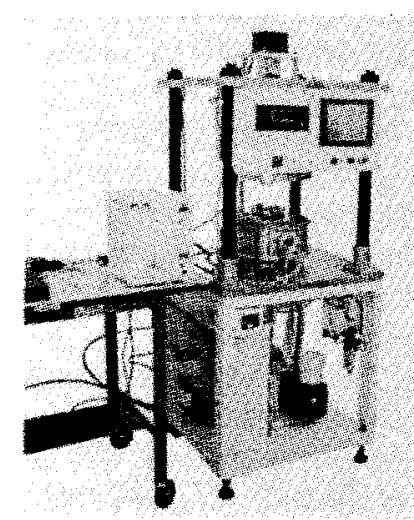

(a)

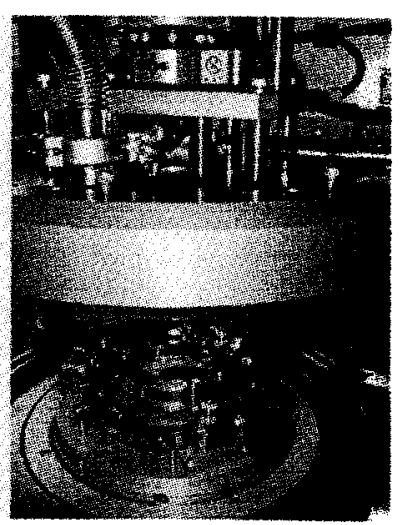

(b)
Figure 1. External view of the imprinting equipment. (LTNIP-5000)

\section{Pre-Exposure Process (PEP)}

The following chart illustrates the procedures for the PEP method that we propose in this paper, compared with the conventional UV imprint process.
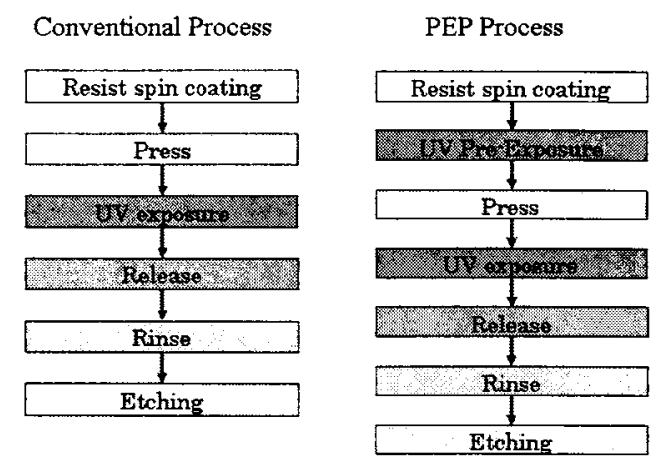

Figure 2. Comparison between the conventional process and the Pre-Exposure Process.

In the conventional UV imprint process, the resin is pressed immediately after the resist coating and exposed to light; however, in the PEP method, 
the resin is subjected to extremely weak exposure prior to the pressing operation so that the resist is slightly hardened. Then, as in the case of the conventional imprint method, the resin is pressed with the mold and subjected to the main exposure with UV light. Figure 3 illustrates the concepts underlying the conventional UV imprinting and PEP methods. In conventional UV imprinting, a coating of resin is applied to the substrate and pressed with a mold while exposed to ultraviolet light for curing. Here, the mold is pressed to the resin with the resin spin-coated (without baking after coating). Since the resin is in liquid form, it conforms to the mold pattern even under low pressure. However, because the resin is liquid, it also readily assumes the configuration of the mold (Figure 3 (a)). In the PEP method, the resin is subject to slight pre-exposure before the mold is pressed, hardening the resin slightly. The viscosity of the resin at this stage is similar to that of the resist softened at or above $T_{g}$ in thermal imprinting. When the mold is pressed against the resin, followed by the exposure to ultraviolet light, the surface is leveled by the mold, and a satisfactory pattern is formed free of interference patterns (Figure 3 (b)).
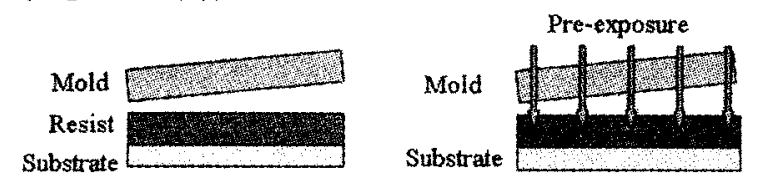

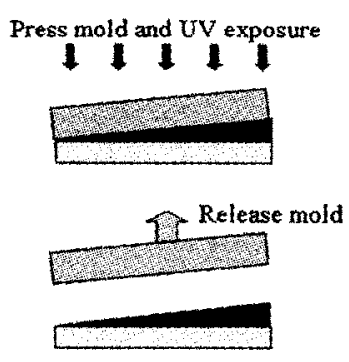

Conventional

(a)

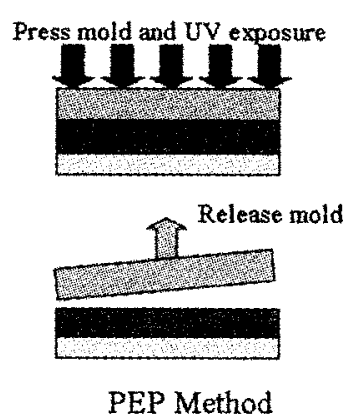

(b)
Figure 3. Schematic diagrams representing the conventional UV imprint method and the PEP method. (a) Conventional (b) PEP method

\section{Experiment and Discussion}

\subsection{Method of experiment}

The resist used in this study was PAK-01 made by Toyo Gosei Co., Ltd. PAK-01 is a resin for UV imprinting of the radical polymerization type based on acrylic resin. Table 1 shows the conditions of the resist process used in this study.
Table-1 Experimental Conditions

\begin{tabular}{ll}
\hline Resist & PAK-01(Toyo Gosei Kogyo) \\
\hline Type & $\begin{array}{l}\text { Acrylic resin of the radical } \\
\text { polymerization type }\end{array}$ \\
\hline Pre-Bake & Not performed \\
\hline Thickness & $4500 \mathrm{~nm}$ (After coating) \\
\hline Exposure & Broad light (300-400nm) \\
\hline
\end{tabular}

First, the change in functional groups during exposure, which corresponds to the crosslinking reaction, was measured to determine the relationship between light exposure and the crosslinking ratio, using FT-IR equipment [11] provided with an exposure function. Then, experimental UV imprinting was performed using a quartz mold without a pattern by changing the amount of Pre-Exposure, and the thickness distribution of residual films corresponding to each exposure was measured in order to determine the optimal amount of Pre-Exposure for providing the desired thickness distribution of the films that does not generate interference patterns. Finally, experimental imprinting was performed with the optimal amount of Pre-Exposure using a quartz mold with a pattern, and the transcription accuracy was compared with that obtained through conventional UV imprinting, in which PreExposure is not performed, in order to verify the effects of the Pre-Exposure Process.

\subsection{Measurement of the crosslinking ratio using FT-IR system}

Figure 4 shows the photo of the crosslinking reaction Analyzer PAGA-100 (LTJ). Figure 5 (a) shows the scheme of the crosslinking reaction of PAK-01, and Figure 5 (b) shows the results of measurements using the crosslinking reaction analyzer. As the photo-reaction initiator is decomposed by the UV exposure, PAK-01 generates free radicals. The free radicals bring about acrylic polymerization, and the polymerization reaction of unsaturated oligomers increases the number of vinyl groups. Thus, it is possible to obtain the relationship between exposure dose and the crosslinking ratio of the resin by measuring the change in the quantity of vinyl groups while the resin is exposed to UV light. 


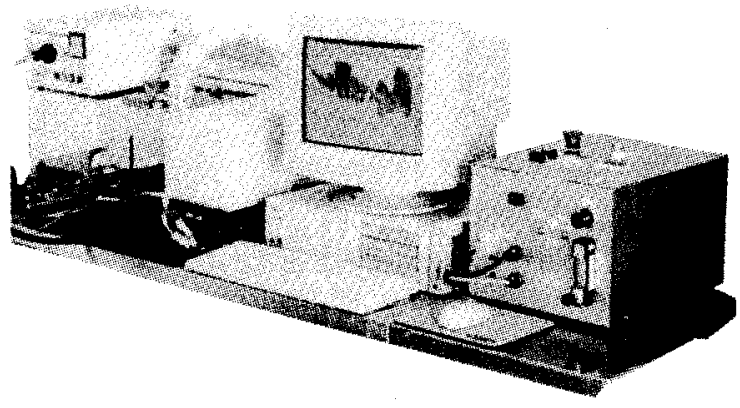

Figure 4 Crosslinking reaction analyzer.

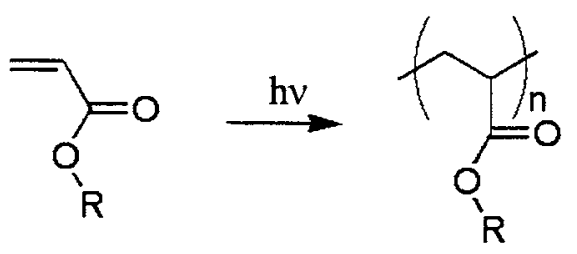

(a)

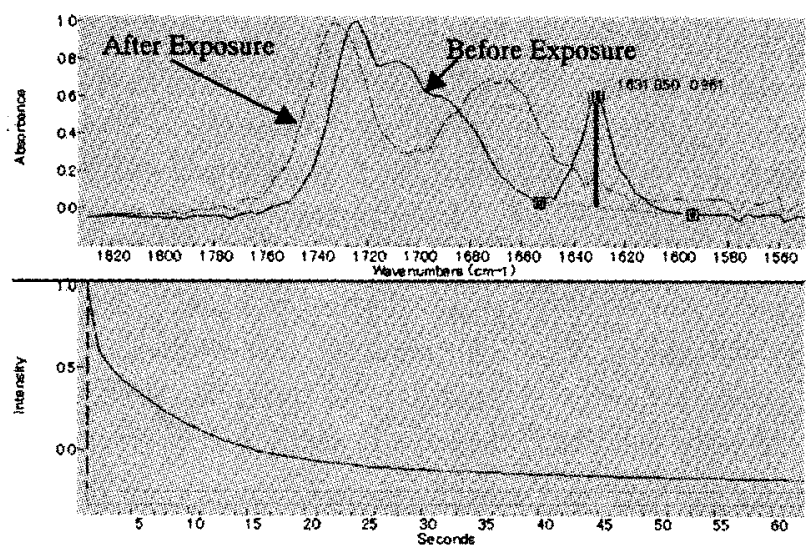

(b)

Figure 5. (a) Reaction scheme of the crosslinking reaction of PAK-01 and (b) Spectra before and after the exposure (top) and the change in absorption due to the functional group at $\mathbf{1 6 3 2}$ $\mathrm{cm}^{-1}$ (bottom).

The fact that the spectral absorption increases at around $1632 \mathrm{~cm}^{-1}$ indicates that the light exposure caused the crosslinking reaction of functional groups. As the irradiation energy is $1 \mathrm{~mW} / \mathrm{cm}^{2}$, the exposure per second is $1 \mathrm{~mJ} / \mathrm{cm}^{2}$. By taking the peak area before exposure as zero crosslinking and the peak area after exposure as 100 crosslinking, the relationship between exposure and the crosslinking ratio is obtained as shown in Figure 6.

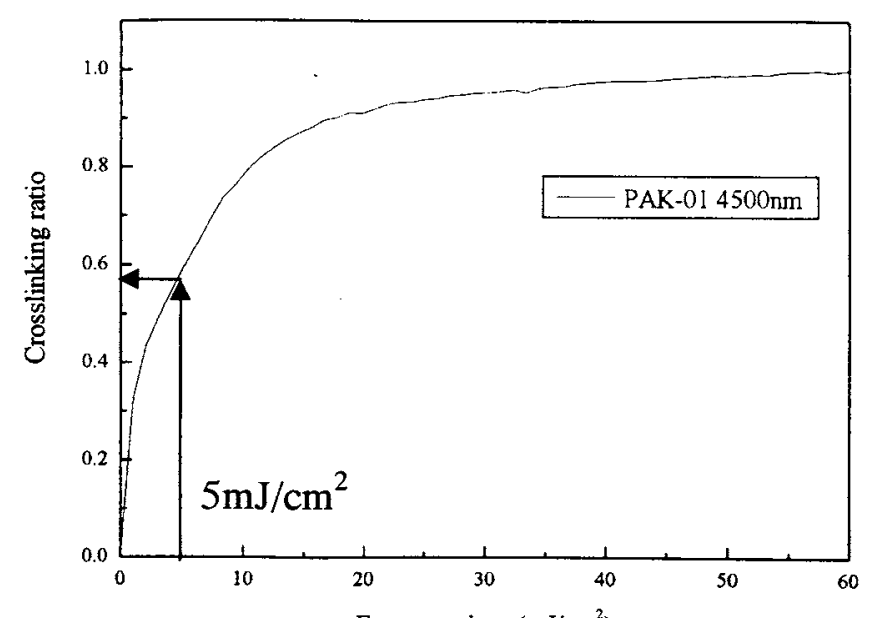

Figure 6. Relationship between the crosslinking ratio and exposure dose.

Figure 6 shows that, taking the crosslinking ratio obtained at $60 \mathrm{~mJ} / \mathrm{cm}^{2}$ as $100 \%$, the crosslinking ratio obtained at an exposure of $10 \mathrm{~mJ} / \mathrm{cm}^{2}$ was approximately $80 \%$, and that obtained at an exposure of $20 \mathrm{~mJ} / \mathrm{cm}^{2}$ was approximately $90 \%$. From this result, it is expected that exposure from 1 to $5 \mathrm{~mJ} / \mathrm{cm}^{2}$ will provide a crosslinking ratio between approximately from $0 \%$ to $58 \%$. As the softness that is required for the resist was likely to be lost with a linking ratio of $58 \%$ or higher, it was decided to perform the Pre-Exposure at 5 $\mathrm{mJ} / \mathrm{cm}^{2}$ or less.

\subsection{Effects of the Pre-Exposure Process}

The effects of pre-exposure are examined using a flat quartz mold without a pattern. The imprinting experiment is performed at pre-exposure doses of 0 $\mathrm{mJ} / \mathrm{cm}^{2}, 1 \mathrm{~mJ} / \mathrm{cm}^{2}, 3 \mathrm{~mJ} / \mathrm{cm}^{2}$, and $5 \mathrm{~mJ} / \mathrm{cm}^{2}$, with pressure set to $1.5 \mathrm{MPa}$ and primary exposure set to $700 \mathrm{~mJ} / \mathrm{cm}^{2}$. (a)

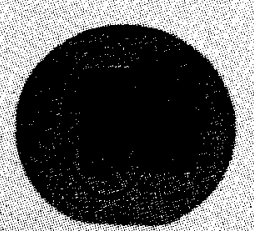

Pre-exposure $=0 \mathrm{~mJ} / \mathrm{em}^{2}$

(c)

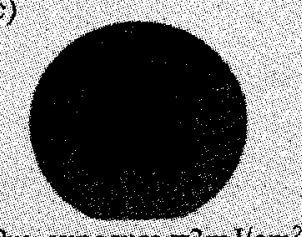

Pre-exposure $=3 \mathrm{~mJ} / \mathrm{cm}^{2}$ (b)

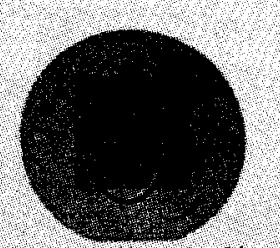

Pre-exposure $=1 \mathrm{~m} / / \mathrm{cm}^{2}$

(d)

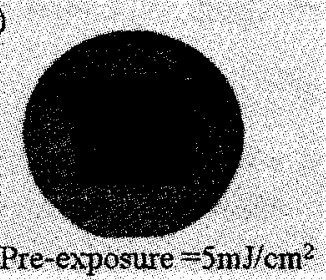

Figure 7. Effects of Pre-Exposure 
Figure 7 shows the imprinting results (based on an observation of residual film distribution) for preexposure doses of $0 \mathrm{~mJ} / \mathrm{cm}^{2}, 1 \mathrm{~mJ} / \mathrm{cm}^{2}, 3 \mathrm{~mJ} / \mathrm{cm}^{2}$, and $5 \mathrm{~mJ} / \mathrm{cm}^{2}$. Without pre-exposure $\left(0 \mathrm{~mJ} / \mathrm{cm}^{2}\right)$, interference patterns appear (circled in Figure 7 (a)), indicating that the residual film distribution is unsatisfactory. With a pre-exposure dose of 1 $\mathrm{mJ} / \mathrm{cm}^{2}$, slight interference patterns still appear at the center. A pre-exposure dose of $3 \mathrm{~mJ} / \mathrm{cm}^{2}$ results in cross-linking and increases resist viscosity, stabilizing residual film distribution and eliminating interference patterns. Figure 8 shows residual film distribution after pressing. The measurement is performed at 49 points set to equidistant intervals of $3.0 \mathrm{~mm}$ in a $25 \mathrm{~mm} \times 25$ mm square area.
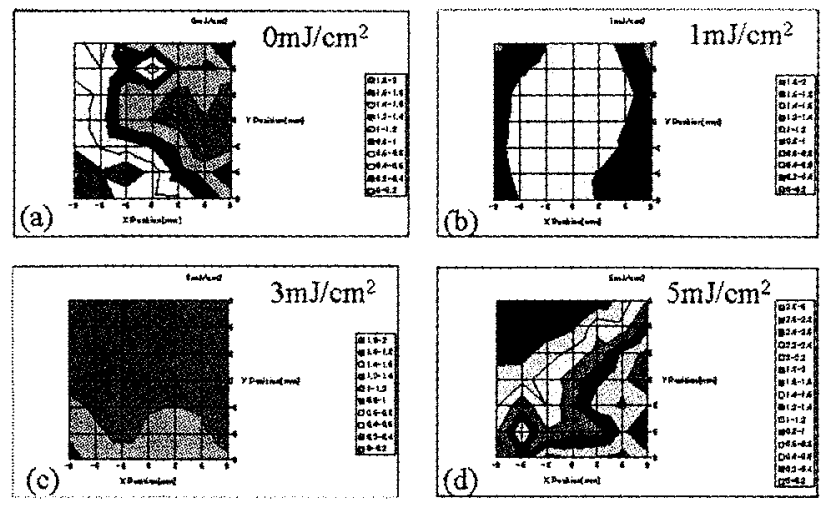

Figure 8. Thickness distribution of residual film: (a) $0 \mathrm{~mJ} / \mathrm{cm}^{2}$, (b) $1 \mathrm{~mJ} / \mathrm{cm}^{2}$, (c) $3 \mathrm{~mJ} / \mathrm{cm}^{2}$, and (d) $5 \mathrm{~mJ} / \mathrm{cm}^{2}$

Table-2 Residual thickness and Pre-exposure dose

\begin{tabular}{|c|c|c|c|c|c|}
\hline Pre-Expo & $0 \mathrm{~mJ} / \mathrm{cm}^{2}$ & $1 \mathrm{~mJ} / \mathrm{cm}^{2}$ & $3 \mathrm{~mJ} / \mathrm{cm}^{2}$ & $\mathbf{4 m J} / \mathrm{cm}^{2}$ & $\mathbf{5 m J} / \mathrm{cm}^{2}$ \\
\hline Min & 0.1423 & 0.6825 & 0.8890 & 1.2460 & 1.1356 \\
\hline Max & 1.4193 & 1.0847 & 1.3937 & 2.2776 & 2.6051 \\
\hline R & 1.2770 & 0.4022 & 0.5048 & 1.0316 & 1.4695 \\
\hline Average & 0.8289 & 0.8125 & 1.2509 & 1.7268 & 1.9567 \\
\hline SD & 0.3330 & 0.1062 & 0.1068 & 0.2752 & 0.4396 \\
\hline
\end{tabular}

Unit: $\mu \mathrm{m}$

The minimum thickness without pre-exposure $\left(0 \mathrm{~mJ} / \mathrm{cm}^{2}\right)$ is $0.142 \mu \mathrm{m}$, while maximum thickness is $1.42 \mu \mathrm{m}$, resulting in a film thickness variation of $1.2 \mu \mathrm{m}$ across the range. The standard deviation for in-plane film thickness distribution is 0.333 . For a pre-exposure of $1 \mathrm{~mJ} / \mathrm{cm}^{2}$, minimum thickness is $0.68 \mu \mathrm{m}$ and maximum thickness is $1.08 \mu \mathrm{m}$, for a range of $0.40 \mu \mathrm{m}$. The standard deviation for in- plane film thickness distribution is 0.106 , approximately one-third that for $0 \mathrm{~mJ} / \mathrm{cm}^{2}$. The results for $3 \mathrm{~mJ} / \mathrm{cm}^{2}$ are similar. For $4 \mathrm{~mJ} / \mathrm{cm}^{2}$, residual film thickness is large, with a minimum thickness of $1.25 \mu \mathrm{m}$ and a maximum thickness of $2.28 \mu \mathrm{m}$, a range of $1.0 \mu \mathrm{m}$. The results for 5 $\mathrm{mJ} / \mathrm{cm}^{2}$ indicate that resist viscosity increases and increases residual film thickness. Figure 9 shows the relationship between the pre-exposure dose and average residual thickness.

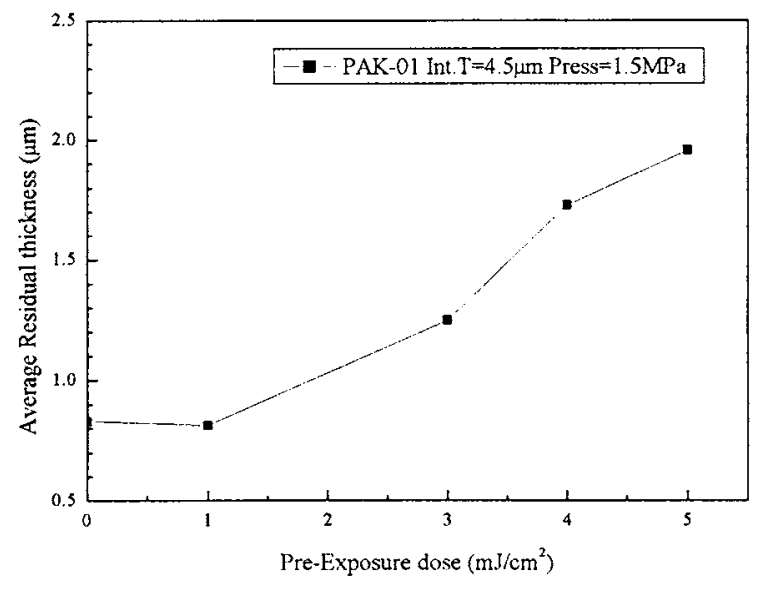

Figure 9. Relationship between pre-exposure dose and average residual thickness.

The results show that a pre-exposure dose of 3 $\mathrm{mJ} / \mathrm{cm}^{2}$ produces adequate pre-exposure effects for PAK-01. Since no interference patterns are observed at $3 \mathrm{~mJ} / \mathrm{cm}^{2}$ and in-plane fluctuations (standard deviation) are also small at this dose, the optimum pre-exposure dose is determined to be 3 $\mathrm{mJ} / \mathrm{cm}^{2}$. With this dose, a UV imprinting experiment is performed by the PEP method using a patterned mold. Another UV imprinting experiment is performed without pre-exposure $\left(0 \mathrm{~mJ} / \mathrm{cm}^{2}\right)$, likewise using a patterned mold, and the pattern transfer results are compared. A quartz glass mold with a raised pattern including L/S of 160 to 400 $\mathrm{nm}$ was used in the experiment. As the height of the pattern was $1 \mu \mathrm{m}$, the aspect ratio of the minimum line width of $160 \mathrm{~nm}$ was 6.25 . UV imprinting experiments were conducted without Pre-Exposure and with Pre-Exposure of $3 \mathrm{~mJ} / \mathrm{cm}^{2}$ using silicon wafers $35 \mathrm{~mm}$ square.

The same mold was used for both conditions, and the pressing pressure and main exposure were $1.5 \mathrm{M} \mathrm{Pa}$ and $700 \mathrm{~mJ} / \mathrm{cm}^{2}$, respectively. Figure 10 shows photos of the substrates after patterning. 


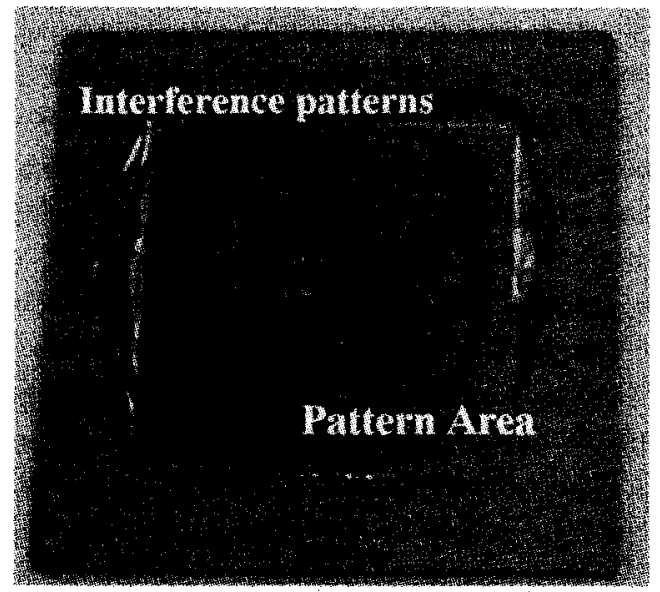

(a)

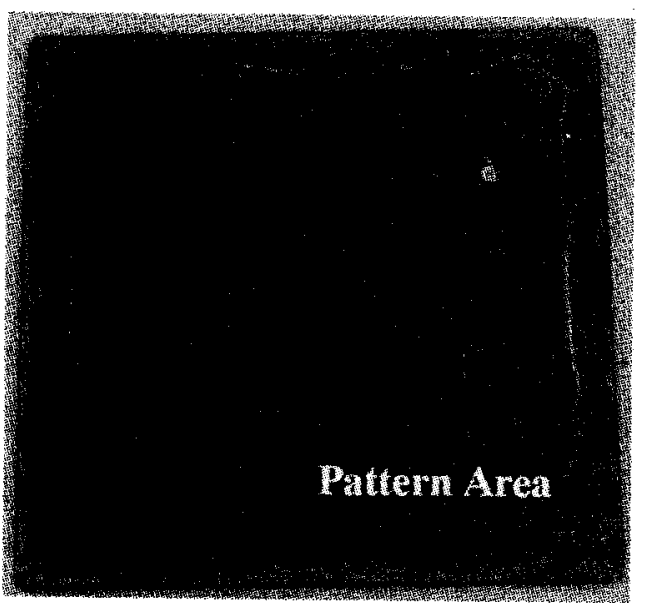

(b)

Figure 10. Comparison between the substrates after patterning as prepared by the conventional method and the PEP method

(Pre-Exposure $=3 \mathrm{~mJ} / \mathrm{cm}^{2}$ ).

(a) Without pre-exposure (b)With pre-exposure

As in the case of the experiments without a pattern, when Pre-Exposure was not performed, interference patterns were observed as shown in the photo on the top (circled in Figure 10 (a)). In contrast, with Pre-Exposure of $3 \mathrm{~mJ} / \mathrm{cm}^{2}$, satisfactory patterns without interference patterns were obtained.

\section{Discussion}

To what extent does the resin harden with a preexposure of $3 \mathrm{~mJ} / \mathrm{cm}^{2}$. We measured the relationship between exposure dose and resin viscosity by the QCM technique. [12] When a coating of the sample material is applied to a quartz oscillator and a voltage is applied, the change in thickness of the sample (resist) is reflected in the change in resonance frequency of the oscillator. Thus, changes in resonance frequency can be used to determine changes in sample film thickness. Since mechanical loss (change in viscoelasticity) in the sample appears as a change in impedance, changes in impedance can be used to determine changes in the mechanical loss of the sample. [13] Approximately $4500 \mathrm{~nm}$ of PAK-01 is applied to a QCM substrate. Figure 11 (a) shows the photo and design of measurement system.

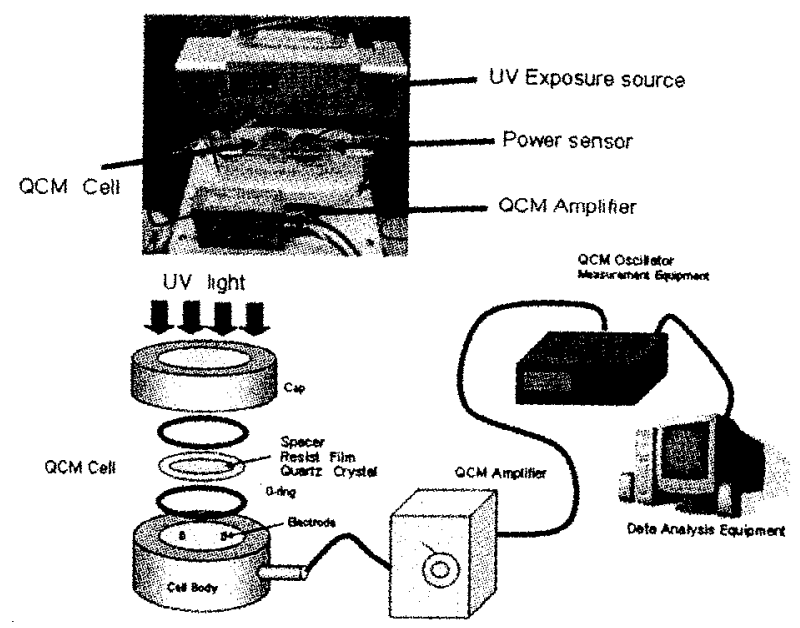

(a)

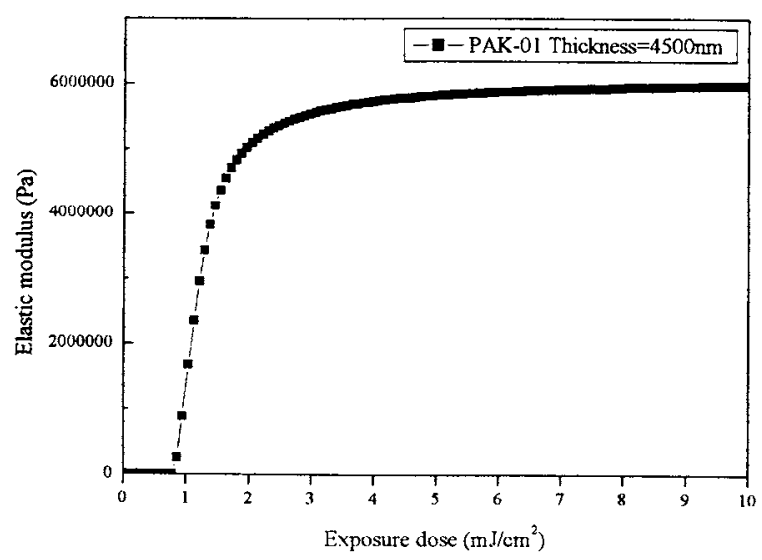

(b)

Figure 11. (a) QCM measurement system. (b) Relationship between elastic modulus and exposure dose.

Figure 11 (b) shows the relationship between the elastic modulus of the resin and exposure dose. The elastic modulus of unexposed PAK-01 is 29.9 Pa. As the exposure dose increases, the elastic modulus decreases until the exposure dose reaches approximately $0.8 \mathrm{~mJ} / \mathrm{cm}^{2}$, then increases sharply. The elastic modulus at the exposure dose of $3 \mathrm{~mJ} / \mathrm{cm}^{2}$, the 
pre-exposure dose determined to be optimal in this study, is $5.54 \mathrm{MPa}$. The crosslinking ratio at this dose is 48 percent when measured by FT-IR. This condition is optimal for preventing interference patterns. In contrast, the elastic modulus at the exposure dose of $1 \mathrm{~mJ} / \mathrm{cm}^{2}$ is 1.68 $\mathrm{MPa}$. In this state, the resin remains close to liquid form, which explains the emergence of slight interference patterns. The elastic modulus at the exposure dose of $5 \mathrm{~mJ} / \mathrm{cm}^{2}$ is $5.82 \mathrm{MPa}$, a value indicating excessive curing for the preexposure stage.

Figure 12 shows SEM photographs of $160 \mathrm{~nm}$, $200 \mathrm{~nm}$ pattern and $320 \mathrm{~nm}, 360 \mathrm{~nm}$ pattern transcribed by the PEP method; it can be seen from these photographs that satisfactory patterns are obtained by this method.

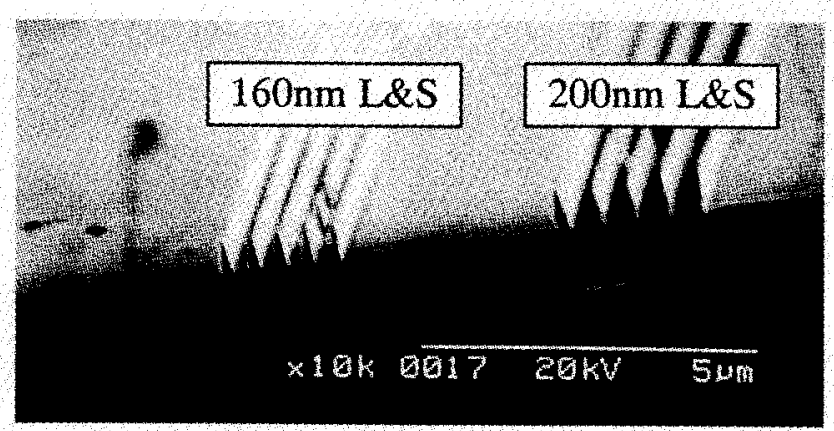

(a)

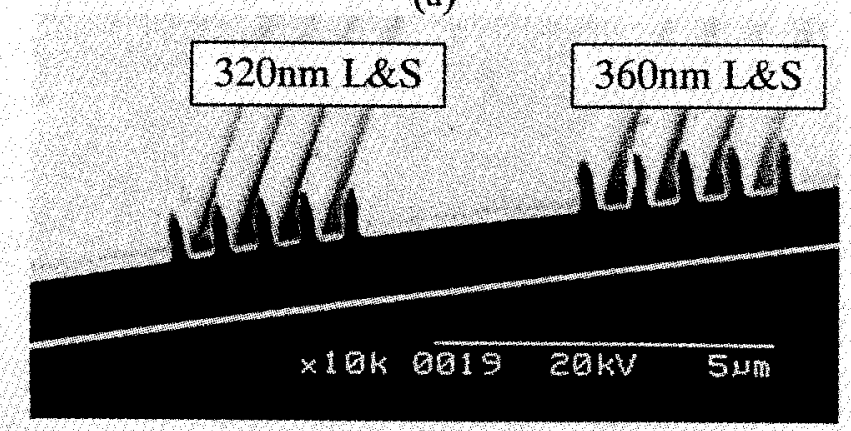

(b)

Figure 12. Patterns transcribed by the PEP method. (a) $160 \mathrm{~nm}$ and $200 \mathrm{~nm} L \& S$ pattern (b) $320 \mathrm{~nm}$ and $360 \mathrm{~nm} \mathrm{~L} \& S$ pattern

\section{Conclusion}

We have attempted to improve the thickness distribution of residual films, which is one of the problems relating to the UV imprint technology, using the PEP method. The relationship between light exposure and the crosslinking ratio was investigated using Crosslinking reaction analyzer
(FT-IR equipment with exposure tool) provided with an exposure function (PAGA-100 made by LTJ) to determine the optimum Pre-Exposure, and experiments on UV imprinting were then conducted using a mold without a pattern. It was confirmed from the results of the study that the PEP method is effective in improving the thickness distribution of residual films, and that the PEP method provides satisfactory patterning.

\section{Acknowledgements}

The authors would like to express their appreciation to Mr. Nobuji Sakai, Mr. Makoto Otaguchi and Tamano Hirasawa of Photosensitive Material Research Center, Toyo Gosei Co., Ltd. for the materials and helpful advice they have provided. The authors would like to express their appreciation to Mr. Naruo Arasaki and Mr. Koichi Hattori of Parusa Corporation design of imprint system LTNIP-5000 too.

\section{Reference}

[1] S. Y. Chou, P. R. Kreauss and P. J. Renstom: Appl. Phys. Lett. 67, 3114 (1996).

[2] P. R. krauss and S. Y. Chou: J. Vac. Sci. Technol. B13, 2850 (1995).

[3] S. Y. Chou, P. R. Krauss, W. L. Guo and L. Zhuang: J. Vac. Sci. Technol. B15 2897 (1997).

[4] L. Guo, P. R. Krauss and S. Y. Chou: Appl. Phys. Lett. 17, 1881 (1997).

[5] Y. Kurashima, et al., Jpn. J. Appl. Phys. 42, 3871 (2003).

[6] Y. Hirai, T. Konishi, T. Yoshikawa, and S. Yoshida: J. Vac. Sci. Technol. B22, 3288 (2004).

[7] T. Bailey, B. J. Choi, M. Colburn, M. Meissi, S. Shaya, J. G.Ekerdt, S. V. Sreenivasan and C. G. Willson: J. Vac. Sci. Technol. B18, 3572 (2000).

[8] M. Komuro, J. Taniguchi, S. Inoue, N. Kimura, Y. Tokano, H. Hiroshima and S. Matsui: Jpn. J. Appl. Phys. 39,7075 (2000).

[9] M. Colburn, T. Bailey, B. J. Choi, J. G.Ekerdt, S. V. Sreenivasan and C. G. Willson: Solid State Technol., 67 (2001).

[10] Y. Hirai, H. Kikuta, and T. Sanou: J. Vac. Sci. Technol. B21, 2777 (2003).

[11] A. Sekiguchi, Y. Miyake, and M. Isono: Jpn. J. Appl. Phys. 391392 (2000).

[12] G. Sauerbrey, Z.f. Phys., 155, 206 (1959).

[13] K.S. Van Dyke, Proc. I.R.E., 16, 742 (1928). 